\title{
A high-fat diet is associated with altered adipokine production and a more aggressive esophageal adenocarcinoma phenotype in vivo
}

\author{
Aaron J. Fowler, BS, Amanda L. Richer, BS, Ross M. Bremner, MD, PhD, and Landon J. Inge, PhD
}

\section{ABSTRACT}

Objective: Obesity has been linked to esophageal adenocarcinoma (EAC). We hypothesize that adipokines, which are altered by obesity, could affect EAC growth rates and potentially serve as biomarkers of disease and targets for treatment. We have developed a potential murine model to investigate the effects of obesity-altered adipokines on EAC in vivo.

Methods: Severe combined immune-deficient mice were fed either a high-fat diet (HFD) containing $60 \%$ animal fat, or a control diet with $10 \%$ animal fat, and monitored for weight gain for 5 weeks. All mice were subcutaneously implanted with EAC cells (OE33), and tumor volume was monitored for an additional 4 weeks by direct measurement and uptake of fluorescently labeled 2-D-deoxyglucose. At sacrifice, serum triglyceride levels and abdominal fat-pad weight were measured to assess obesity state. Adipokine levels were measured within abdominal fat of tumor-bearing mice.

Results: Mice fed the HFD displayed increased body weight, visceral fat, and serum leptin and triglycerides. All mice developed tumors; OE33 EAC cells in HFD mice displayed increased growth rates, proliferation, and metabolic activity relative to tumors of EAC in control diet mice. Adipokine expression in the abdominal fat revealed distinct changes associated with the HFD and increased body weight.

Conclusions: Ad libitum feeding of the HFD was correlated with moreproliferative EAC tumors in vivo. This phenotype was associated with alterations to secreted adipokines, representing a potential mechanism for our observations. Further studies are necessary to explore findings, as they have potential to improve treatment of EAC. (J Thorac Cardiovasc Surg 2015;149:1185-91)

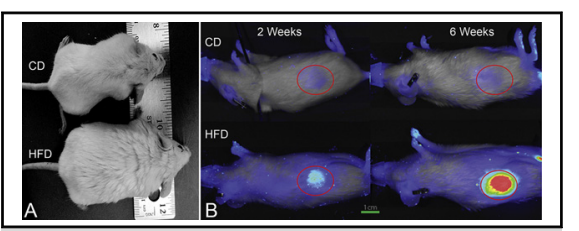

(A) A diet high in fat (HFD) in SCID mice correlates with increased tumor growth (B) compared with SCID mice fed a standard, control $\operatorname{diet}(C D)$.

\section{Central Message}

Ad libitum feeding of the high-fat diet correlated with more proliferative EAC tumors in vivo. This phenotype was associated with alterations to secreted adipokines, representing a potential mechanism for our observations. Further studies are necessary to explore findings as they have potential to improve treatment of EAC.

\section{Perspective}

EAC is one of the few malignancies with a rising incidence. Obesity is a primary risk factor for EAC; however, little is known as to how obesity contributes to EAC pathogenesis. Suitable preclinical models to study this association are lacking. We find that ad libitum feeding of a highfat diet results in increased growth of a human EAC in SCID mice, and mice display characteristics associated with obesity induced metabolic disease. Our study describes a preclinical, in vivo platform that may provide insight into the association between obesity and EAC and for developing potential treatments for EAC.

See Editorial Commentary page 1192
The incidence of esophageal adenocarcinoma (EAC), a disease with an overall survival of only $17 \%,{ }^{1}$, has risen by more than $300 \%$ over the past 25 years, ${ }^{2}$ necessitating identification of risk factors that contribute to this disease. Over the same time period, obesity rates in the United States and worldwide have risen dramatically, doubling in the United States since 1980, with more than half of the population predicted to be obese within

\footnotetext{
From the Norton Thoracic Institute, St. Joseph's Hospital and Medical Center, Phoenix, Ariz.

This work was funded by grants from the St. Joseph's Foundation (Phoenix, Ariz) and the American Lung Association (RG-224607-N-L.J.I)

Read at the 40th Annual Meeting of The Western Thoracic Surgical Association, Dana Point, California, June 25-28, 2014.

Received for publication June 26, 2014; revisions received Nov 18, 2014; accepted for publication Nov 28, 2014; available ahead of print Jan 13, 2015.

Address for reprints: Landon J. Inge, PhD, Norton Thoracic Institute, St. Joseph's

Hospital and Medical Center, 445 N 5th St, Suite 110, Phoenix, AZ 85004

(E-mail: landon.inge@ dignityhealth.org).

$0022-5223 / \$ 36.00$

Copyright (c) 2015 by The American Association for Thoracic Surgery

http://dx.doi.org/10.1016/j.jtcvs.2014.11.076
}

the next 10 years. ${ }^{3}$ Epidemiologic studies have linked obesity to several types of cancer, and it is a recognized risk factor for EAC. ${ }^{4-7}$ The mechanisms behind this association remain unclear and complex. Understanding how molecular pathways altered by obesity can affect EAC would offer new opportunities for prevention and treatment of this disease.

Previous in vitro and in vivo laboratory studies have noted a relationship between obesity and increased tumor growth rates and disease progression in breast, colon, and other cancers. $^{4,5,8-10}$ Although the exact mechanism(s) behind this observation is unclear, several studies have focused on the ability of adipose tissue to function as an endocrine organ. ${ }^{11}$ Adipose tissue is capable of releasing bioactive molecules, or adipokines, necessary for normal metabolic functions. Obesity alters the function of adipose cells, causing changes in the expression and secretion of adipokines into the blood. ${ }^{12}$ Several adipokines have tumorigenic effects, altering the in vitro proliferation of various cancer cells. Leptin, a wellstudied adipokine involved in metabolic regulation, 


$$
\begin{aligned}
& \text { Abbreviations and Acronyms } \\
& \text { CD }=\text { control diet } \\
& \text { EAC }=\text { esophageal adenocarcinoma } \\
& \text { HFD }=\text { high-fat diet } \\
& \text { NOD }=\text { nonobese diabetic } \\
& \text { SCID }=\text { severe combined immune-deficient } \\
& \text { 2DG }=2 \text {-deoxyglucose }
\end{aligned}
$$

has been shown to increase proliferation and survival of the OE33 EAC cell-line in vitro ${ }^{13,14}$; increased leptin levels are correlated with increased risk of Barrett's esophagus, ${ }^{15}$ and adiponectin induces apoptosis of an EAC cell-line in vitro. ${ }^{16}$ However, the effects of various adipokines on cancer cells in vivo are more complex, and understanding their effects will require model systems that can more closely mimic the contributing effects of obesity in EAC.

In the present study, a mouse model was developed, to better investigate the effects of obesity and adipokines on EAC. A diet high in fat resulted in characteristics of obesity (significant weight gain, increased visceral body fat, and increased serum triglycerides and leptin) in immune-deficient mice. These changes were correlated with increased growth of a human EAC cell-line implanted in the flank. Analysis of EAC tumors in mice revealed that increased metabolic activity and proliferative index were associated with the high-fat diet (HFD) and obesity measures; studies of adipokine levels in the adipose tissue showed changes specific to mice fed the HFD. These findings suggest a potential model for studying the link between adipokines and EAC.

\section{MATERIALS AND METHODS Cell Culture}

The human EAC cell-line OE33 (Sigma-Aldrich. St Louis, Mo) was maintained in RPMI-1640 (Life Technologies, Grand Island, NY), supplemented with $10 \%$ fetal bovine serum, $1 \mathrm{X}$ nonessential amino acids, and $1 \mathrm{X}$ penicillin streptomycin neomycin (all Life Technologies) and incubated at $37^{\circ} \mathrm{C}$, with $5 \% \mathrm{CO}_{2}$ and $95 \%$ humidity. Notably, OE 33 has been validated as a human EAC cell-line, in a study to determine the origin of several commonly used EAC cell-lines. ${ }^{17}$

\section{In Vivo Modeling and Imaging}

Nonobese, diabetic, severe combined immune-deficient (NOD-SCID [NOD.Cg-Prkdc $\left.{ }^{\text {scid }} \mathrm{Il} 2 \mathrm{rg}^{\mathrm{tm} 1 \mathrm{Wjl}} / \mathrm{Szj}\right]$ ) mice were obtained from Jackson Labs (Bar Harbor, Maine). They were housed, maintained and used under an Institutional Animal Care and Use Committee-approved protocol. Mice ( $n=20$, aged 6-8 weeks) were randomized to a control diet (CD) containing $10 \%$ animal fat (TestDiet, St Louis, Mo, catalog \#58Y2), or an HFD containing $60 \%$ animal fat (TestDiet, catalog \#58Y1). The diets are standard in murine studies of obesity. ${ }^{18}$ Body weights were measured weekly throughout the study.

Ten million OE33 cells were added to a solution of $750 \mu$ l of growth factor-reduced BD Matrigel (BD Biosciences, Franklin Lakes, NJ) and $250 \mu 1$ of RPMI-1640 kept on ice before use. One million cells, in $100 \mu \mathrm{l}$ of the
cell/Matrigel solution, were injected subcutaneously into the right flank after 5 weeks on respective diet (CD or HFD). Palpable tumors ( 2 weeks postinjection) were measured using manual calipers (Roboz Surgical Instrument Company, Gaithersburg, Md), and tumor volumes were calculated as described elsewhere. ${ }^{19}$ For in vivo imaging of OE33 tumors, mice were anesthetized using $2 \%$ isoflurane and injected with $10 \mathrm{nM}$ of 2deoxyglucose (2DG) IRDye (LI-COR, Lincoln, Neb) via the tail vein.

After a 48-hour clearance period, hair was removed from the tumor site, and uptake of IRD800-2DG was visualized using the Pearl Impulse Small Animal Imaging System (LI-COR). Relative light units, representing 2DG uptake, were determined by using Image Studio 3.1 software (LI-COR). Mice were scanned at 2 and 6 weeks postimplantation of OE33 cells. Statistical analysis of tumor volumes and fold changes of relative light units were analyzed using a Student $t$ test in Prism (GraphPad Software, San Diego, Calif). Intra-abdominal fat pads, liver, and kidney tumors were collected when mice were killed, and the tissues were flash-frozen or fixed in $10 \%$ neutral buffered formalin for use in immunohistochemistry.

\section{Cytokine and Adipokine Arrays}

Expression levels of adipokines were analyzed using protein lysates collected from abdominal fat-pad tissue in the same manner as protein used for immunoblotting. Protein $(250 \mu \mathrm{g})$ was loaded onto a Proteome Profiler Mouse Adipokine Array Kit membrane (R\&D Systems, Minneapolis, Minn) and allowed to incubate overnight at $4^{\circ} \mathrm{C}$. The membranes were developed using reagents included in the kit and exposed on the C-digit. Densitometry was completed using ImageJ software (http://imagej.nih. gov/ij/), acquired from the National Institutes of Health. Expression levels of cytokines were analyzed in the same manner, using Proteome Profiler Mouse Array Kit, Panel A, (R\&D Systems).

\section{Assessment of Blood Glucose and Serum Triglyceride and Leptin Levels}

After an overnight fast, baseline blood glucose was measured using a OneTouch Ultra2 monitoring system (LifeScan, Inc, Milpitas, Calif) including OneTouch Ultra Blue Test Strips, according to the manufacturer specifications. Blood was immediately sampled after a tail nick. Readings were taken weekly at the same time of day.

Whole blood was collected in a serum BD Vacutainer (BD BioSciences, Franklin Lakes, NJ) and allowed to clot at room temperature for $30 \mathrm{~min}$. Serum was isolated after centrifugation for $10 \mathrm{~min}$ at $2000 \mathrm{~g}$. Samples were either analyzed immediately or aliquoted and stored at $-80^{\circ} \mathrm{C}$. Triglyceride levels in serum were assessed using the Triglyceride Quantification Kit (abcam, Cambridge, Mass), according to manufacturer specifications. Serum leptin levels were analyzed using the Mouse/Rat Leptin Quantikine ELISA Kit (R\&D Systems), according to manufacturer specifications.

\section{Immunohistochemistry}

Formalin-fixed tissues were processed into paraffin blocks using routine procedures. Sections of $5 \mu \mathrm{m}$ were deparaffinized and rehydrated; heat-induced epitope retrieval was performed as described elsewhere. ${ }^{20}$ Tissues were blocked with $5 \%$ fetal bovine serum in phosphate-buffered saline, and peroxidase activity was abolished by the addition of a $1 \%$ hydrogen peroxide phosphate-buffered saline solution. Primary antibody (Ki-67, Cell Signaling Technology, Inc, Danvers, Mass) was diluted in 5\% fetal bovine serum in phosphate-buffered saline at manufacturer-recommended concentration. A Mach4 Universal HRP-Polymer Detection Kit (Biocare Medical, Concord, Calif) was used to apply HRP (horseradish peroxidase), followed by chromogen. Slides were counterstained with hematoxylin and bluing reagent, dehydrated in graded alcohols, cleared, and mounted. Slides were imaged on an Aperio system (Aperio Spectrum, Leica Biosystems, Buffalo Grove, Ill) at 10X magnification. Proliferation indices (average number of Ki-67 stained cells per high-powered field) were completed by blinded analysis of 5 random images and using ImageJ. 

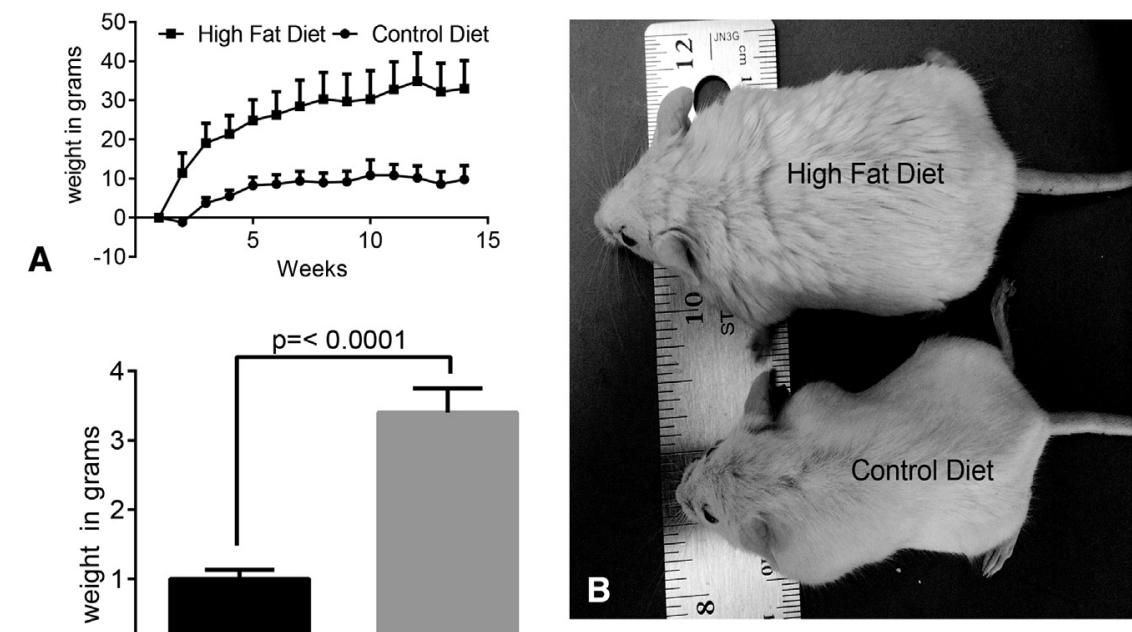

C
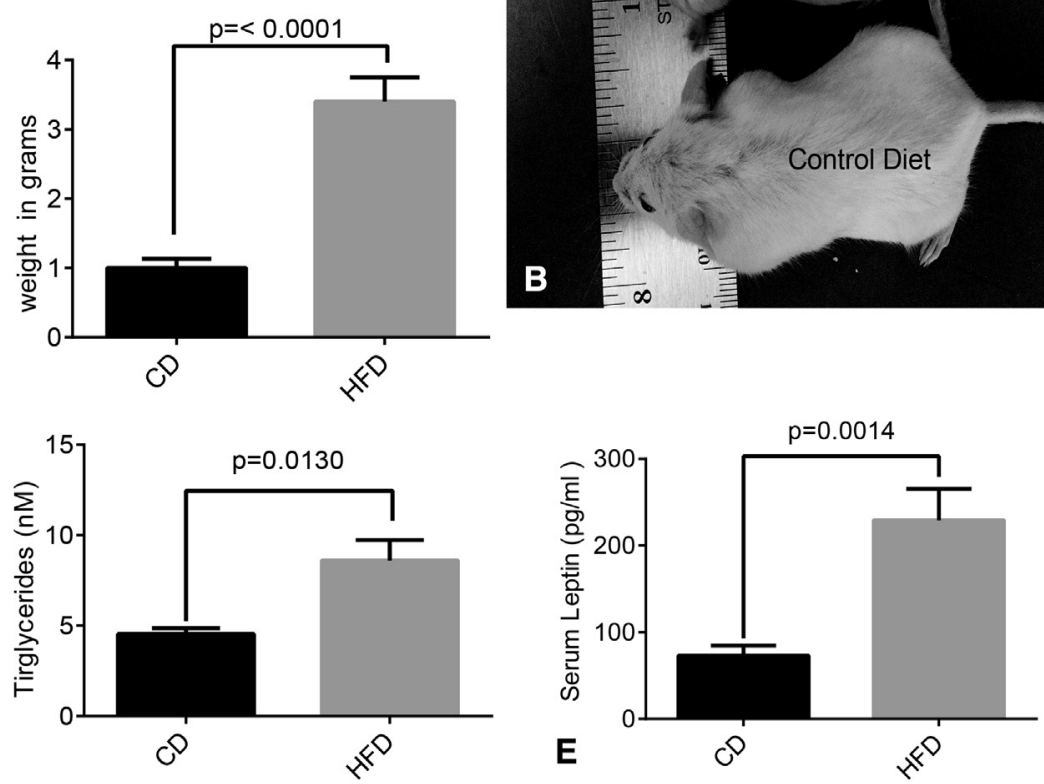

FIGURE 1. An HFD induces characteristics of obesity in NOD.SCID mouse. A, Weight change of NOD.SCID mice fed an HFD ( $\mathrm{n}=10,60 \%$ calories from fat $)$ or $\mathrm{CD}(\mathrm{n}=10 ; 10 \%$ of calories from fat) for 14 weeks. Diet was provided ad libitum, and mice were weighed weekly. Percent weight change was calculated relative to weight upon starting respective diet. Bars represent SE. B, Representative images of NOD.SCID mice after 4 weeks of ad libitum feeding of HFD or CD. C, Weight of abdominal fat pads collected from NOD.SCID mice fed an HFD $(\mathrm{n}=10)$ or CD $(\mathrm{n}=10)$ for 4 weeks. Bars represent SE. Serum levels of triglycerides (D) and leptin (E) in NOD.SCID mice fed an HFD $(\mathrm{n}=10)$ or CD $(\mathrm{n}=10)$ for 4 weeks. Bars represent SE. CD, Control diet; $H F D$, high-fat diet.

\section{RESULTS}

Weight Gain, Tumor Growth, and Metabolism in NOD.SCID Mice That Were Fed Either an HFD or a CD

Based on the link between obesity and EAC, ${ }^{5,21}$ we investigated the effects of dietary fat consumed by NOD.SCID mice, on their body weight and tumor growth, that were fed either a diet containing $10 \%$ animal (lard) fat (CD) or $60 \%$ animal fat (HFD) ad libitum. Several mouse strains have been shown to display distinct obesity characteristics when fed a diet rich in fat. ${ }^{22}$ Specifically, strains prone to obesity (eg, C57BL/J6) display increased weight gain, heavier abdominal fat pads, and increased serum levels of leptin and triglycerides when fed an HFD, relative to mice fed a $10 \%$-fat diet. ${ }^{22}$ NOD.SCID mice that were fed the HFD displayed increased body weight (Figure 1, A), heavier abdominal fat pads (Figure 1, $B$ ), larger body size (Figure 1,C), and increased serum levels of triglycerides (Figure 1,D) and leptin (Figure 1,E). Glucose levels were somewhat higher in NOD.SCID mice on the HFD, but this increase was not statistically significant (data not shown).

Five weeks after introduction of the CD or HFD, 1 million OE33 EAC cells were implanted into the right flank. Palpable tumors were measured for 4 weeks. OE33 tumors displayed increased growth and were found to be $53 \%$ larger in mice that were fed the HFD after 4 weeks of growth $(P=.046)$, compared with OE33 tumors in mice on the CD (Figure 1,B). To further investigate the effect of obesity on the growth of tumors in vivo, we performed measurements of glucose uptake using 2DG labeled with an infrared probe and quantifying relative light units, which has been shown to parallel more standard tumor-volume measurements. ${ }^{23}$ Consistent with the observations of increased tumor volume (Figure 2, A), OE33 tumors in mice on the HFD (Figure 2, B) displayed increased uptake of 2DG, relative to OE33 tumors in nonobese mice. Quantification of the change in relative light units between baseline and 4 weeks showed the uptake of 2DG to be 3 -fold higher in mice fed the HFD (fold change: 30.8 vs 10.3, $P=.0438$ ) (Figure 2, $C$ ). 

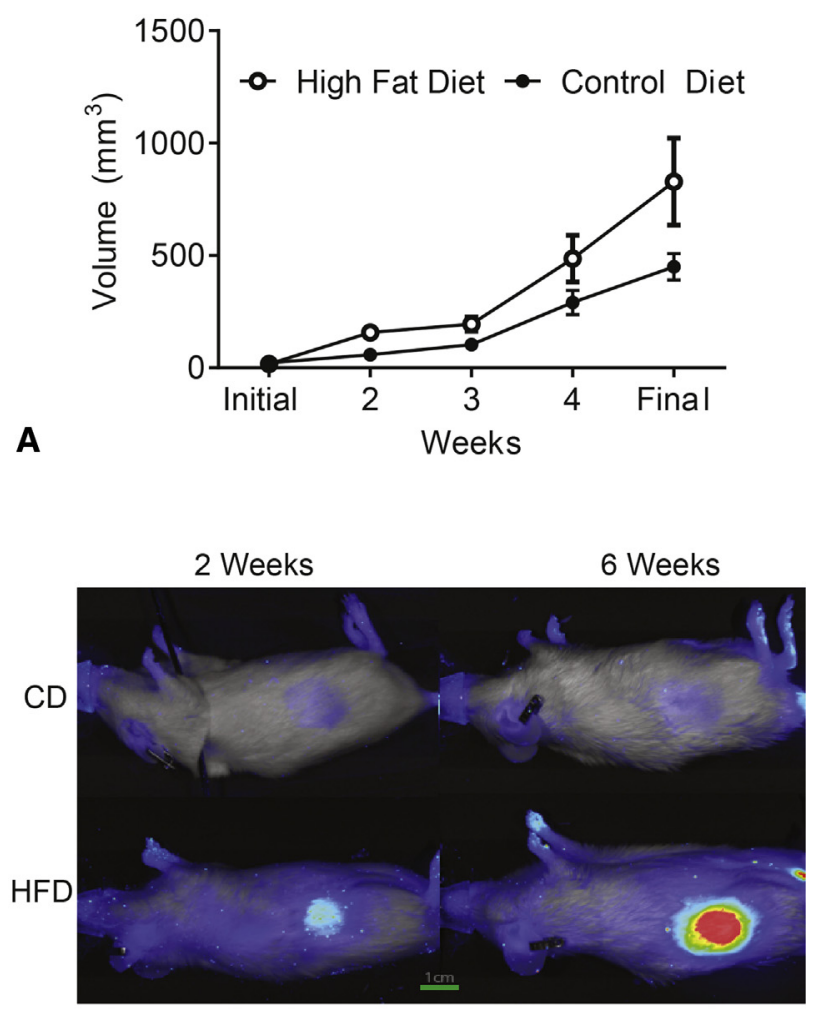

B

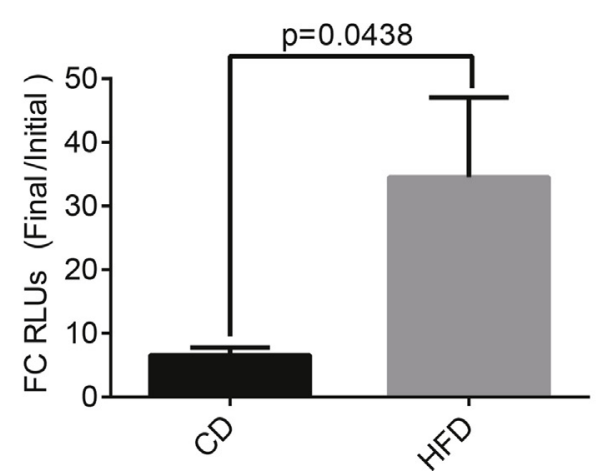

FIGURE 2. HFD correlates with increased growth and size of human EAC tumor xenografts in NOD.SCID mice. A, NOD.SCID mice were implanted with 1 million OE33 EAC cells after ad libitum feeding with an HFD or CD for 4 weeks. At 2 weeks postimplantation, palpable tumors were measured weekly for an additional 4 weeks. Tumor volumes were calculated as described in Materials and Methods. Bars represent SE of 10 independent measurements. B, Representative image of in vivo imaging of fluorescently labeled 2DG in OE33 tumors in mice fed a CD or HFD. C, Quantification of RLUs obtained from in vivo imaging from (A). The fold change was calculated as described in Materials and Methods. Bars represent SE of 10 independent measurements. $C D$, Control diet; HFD, high-fat diet; FC RLU, fold change relative light unit.

\section{Levels of Proliferation Correlate Directly With Dietary Fat}

To further investigate the effects of the HFD on the growth of the OE33 EAC cell-line in vivo, we compared staining of the proliferative marker, $\mathrm{Ki}-67$, in tumors from mice fed the HFD or CD. OE33 tumors were stained by immunohistochemistry, and the proliferative index was assessed by blinded review. Consistent with increased tumor volume and glucose uptake, OE33 tumors in mice on the HFD (Figure 3, $A$ and $B$ ) showed a higher proliferative index, compared with OE33 tumors from mice on the CD (48 cells vs 80 cells per high-power field, $P=.0108$ ).

\section{Expression of Adipokines Correlates With Diet}

In humans, diet-induced obesity results in alterations in expression and secretion of adipokines. ${ }^{12}$ To assess whether ad libitum feeding with the HFD had effects on adipokine secretion and expression in the NOD.SCID strain, we characterized the adipokine profile in the intra-abdominal fat pads of mice on the HFD or CD for 4 weeks (Sham) and mice on the HFD or CD implanted with OE33 xenografts (Tumor). As shown in Figure 4, $A$, mice given ad libitum feeding of the HFD for 4 weeks were found to have higher expression of several adipokines with known proinflammatory and tumorigenic functions (leptin, insulin-like growth factor-binding protein, fibroblast growth factor- $\alpha$ ) (Figure $4, A$ ). In addition, adipose tissue from obese mice displayed decreased expression of adiponectin, CD26, lipocalin, and retinol binding protein 4, reported to have anti-inflammatory and growthsuppressive functions. Mice on the HFD and harboring OE33 xenografts displayed a similar profile within their abdominal fat pads, but they also showed increased expression of TIMP1 (tissue inhibitor of metalloproteinase) and Serpin E1/PAI (plasminogen activator inhibitor) (Figure 4, B), 2 proteins associated with poor prognosis in several malignancies. ${ }^{24-26}$ Of the several adipokines upregulated in our model, leptin has been found to stimulate proliferation of OE33 cells in vitro. ${ }^{13}$ Analysis of serum leptin levels by ELISA (enzyme-linked immunosorbent assay) in CD and HFD mice showed a markedly increased level of leptin in mice on the HFD compared with mice on the CD (Figure 1, $E, 229 \mathrm{vs} 73 \mathrm{pg} / \mathrm{ml}, P=.0014)$.

\section{DISCUSSION}

In the present study, we found that ad libitum feeding of NOD.SCID mice with a diet high in fat resulted in increased weight gain, abdominal fat, serum triglycerides (Figure 1), and serum leptin (Figure 1,E). These characteristics of obesity were correlated with increased growth rates and tumor volumes (Figure 1, B) of the human esophageal adenocarcinoma cell-line, OE33. In the mice on an HFD, OE33 tumors additionally displayed increased tumoral 2DG uptake, as shown by in vivo imaging (Figure 2), and an increased proliferative index, as measured by Ki-67 immunohistochemical staining (Figure 3, A). Metabolic activity, as measured by standardized uptake value in positron emission tomography/computed tomography scanning with the glucose analogue 2-[ $\left.{ }^{18} \mathrm{~F}\right]$ fluoro-2-deoxy-D-glucose, and the Ki-67 index via immunohistochemical staining, 


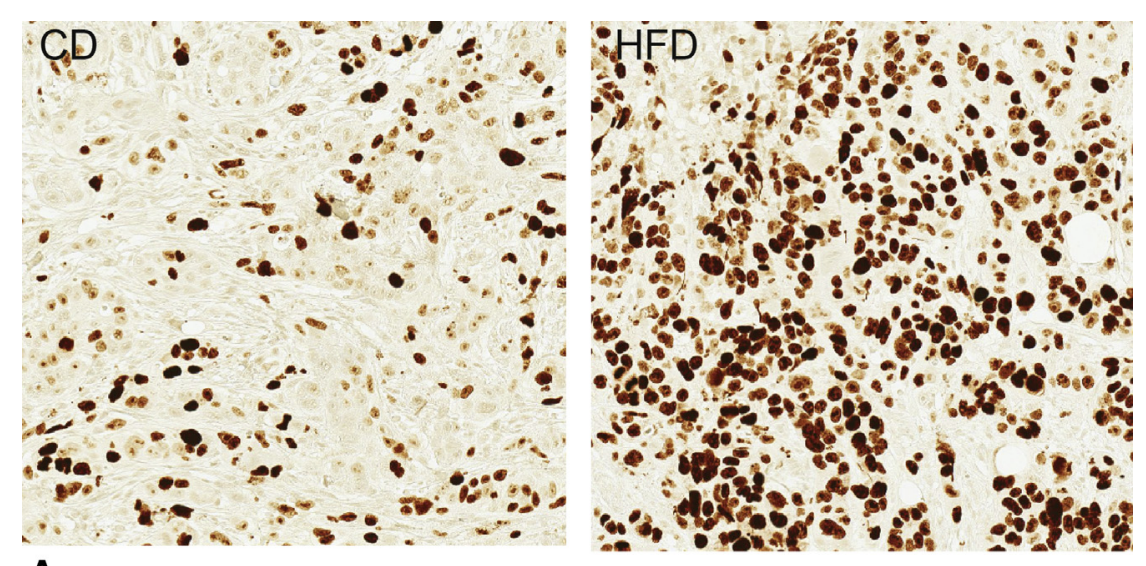

A

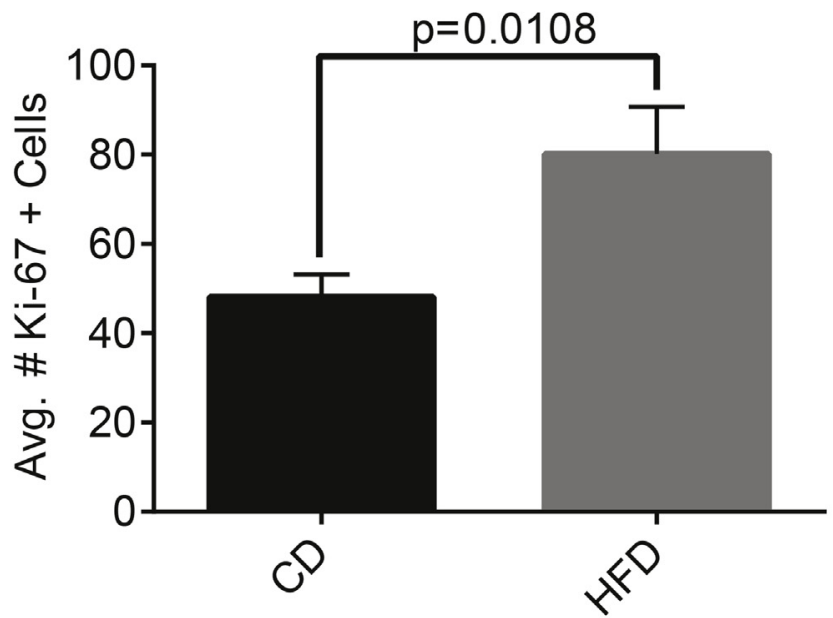

FIGURE 3. OE 33 tumors from obese mice display increased proliferation. A, Representative images of Ki-67 immunohistochemical staining in OE33 tumors from mice fed a CD or HFD. B, Proliferative index of OE33 tumors from mice fed the CD or HFD. Average number of Ki-67 positive cells were calculated as described in Materials and Methods. Data are the average of 5 random fields from 10 mice. Bars represent SE. $C D$, Control diet; $H F D$, high-fat diet; Avg, average.

are used as an indication of the aggressiveness of the tumor. $^{27,28}$ The findings suggest that obesity imparts a more aggressive phenotype on the OE33 EAC cell-line in vivo, consistent with findings in a transgenic colon cancer model $^{29}$ and other solid-tumor models. ${ }^{8,10}$ Adipokine protein levels in abdominal fat pads of HFD mice reveal increased expression of several adipokines known to have roles in cancer, suggesting a possible mechanism for the aggressive growth of the OE33 EAC cell-line in the obese NOD.SCID mouse.

Obesity has been implicated as a risk factor for several cancers of the gastrointestinal tract, including EAC. ${ }^{4}$ Despite the association of obesity with EAC, the mechanisms behind its role in EAC pathogenesis are unclear. In this study, we have attempted to model the effects of obesity on the growth of EAC in vivo. The obese phenotype within obesity-prone mouse strains is well defined. ${ }^{22}$ In particular, ad libitum feeding of a diet high in fat results in increased weight gain, serum triglycerides and leptin, and increased abdominal fat. In this study, ad libitum feeding of an HFD to the NOD.SCID mouse strain resulted in development of these characteristics, suggesting that these mice are prone to obesity. In addition, the presence of this characteristically obese phenotype was correlated with increased tumor volume, tumoral 2DG uptake, and proliferative index, suggesting that the obese state influenced the growth of the OE33 EAC cell-line.

Although the exact mechanism for our observations is currently unknown, chronic background inflammation, a known cofactor of Barrett's esophagus and EAC progres$\operatorname{sion}^{30}$ could be involved. Chronic inflammation results from obesity, ${ }^{12}$ and this effect is shown to be a major driver in a murine model of hepatocellular carcinoma ${ }^{31}$ and is thought to play a role in colon cancer. Although the immune-compromised model used in this study could have dampened inflammation, reported results ${ }^{32}$ show that deficiency in B- and T-cells does not prevent obesityrelated inflammation. However, we recognize that the immune deficiency in our model may complicate translation of findings from our study to human patients. We are 

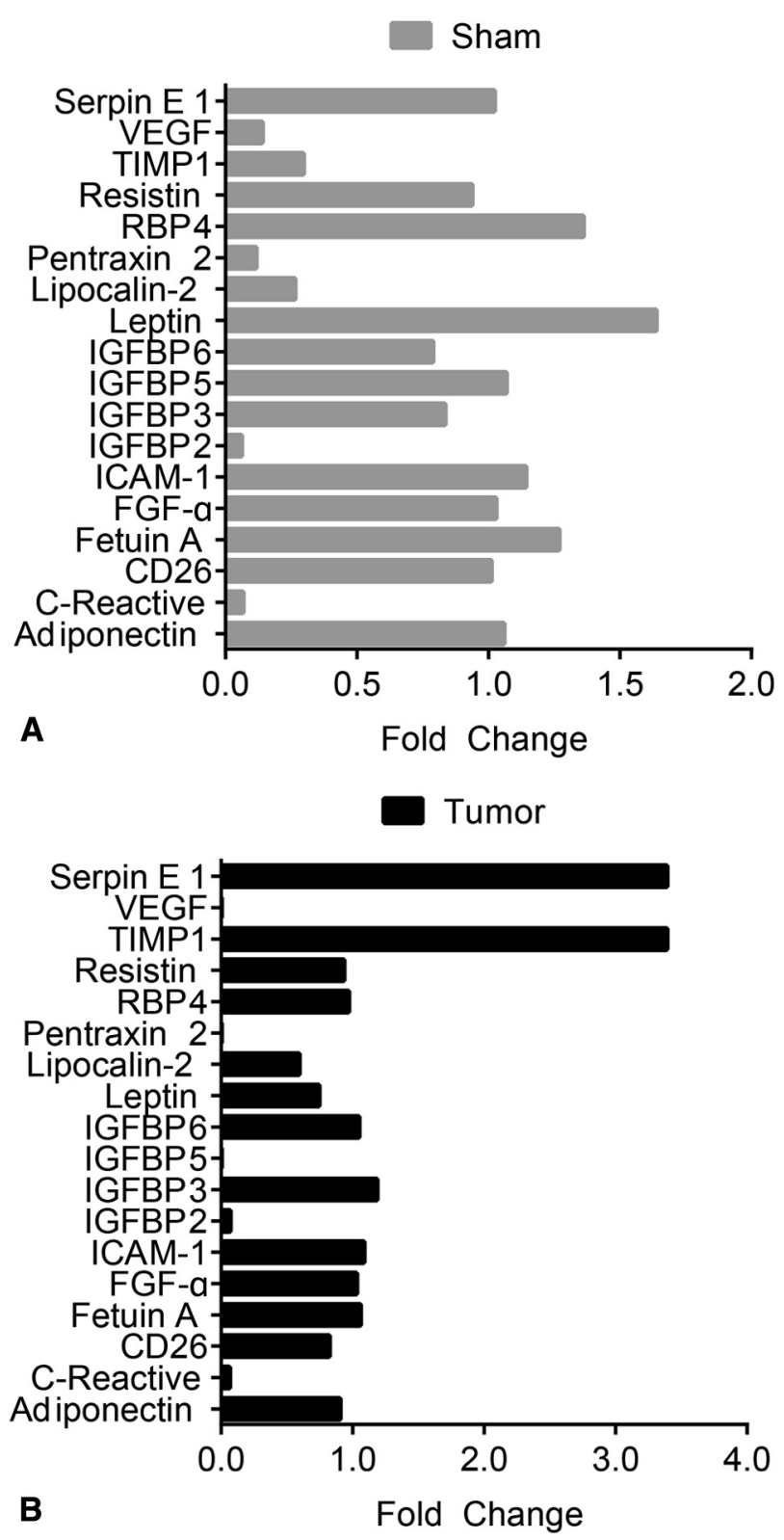

FIGURE 4. Adipokine expression varies as a result of dietary fat consumption and obesity. Abdominal fat pads from mice fed an HFD or CD and implanted with the Sham (A) or human EAC cell-line OE33 (B) were analyzed as described in Materials and Methods. Densitometry analysis of spots using ImageJ were used to generate fold change (HFD/ CD). Presented proteins had the greatest change in expression in HFD mice relative to $\mathrm{CD}$ mice. VEGF, Vascular endothelial growth factor; TIMP, tissue inhibitor of metalloproteinase; $R B P$, retinol binding protein; IGFBP, insulin-like growth factor-binding protein; ICAM, intercellular adhesion molecule; $F G F$, fibroblast growth factor; $C D$, control diet.

currently performing experiments with other EAC celllines, as well as larger animal cohorts, to further validate and improve our findings. In addition, although our study design does suggest that obesity may have an influence on EAC, it cannot elucidate how obesity might contribute to progression of EAC from Barrett's esophagus. Thus, study of the role of obesity in this progression, using more comparable murine models, is critical. A recently developed transgenic murine model of this progression ${ }^{33}$ provides just such means to study this link.

The literature shows a close association between obesity and the incidence of esophageal cancer ${ }^{5,21}$; however, the relationship between body mass index and patient outcome in EAC is unclear, ${ }^{34}$ despite the fact that body mass index is indicative of poor prognosis in colon cancer. ${ }^{35}$ This difference may be related to the observation that central adiposity seems to be more closely associated with esophageal cancer. ${ }^{5}$ Data indicate that, unlike peripheral adipose tissue, visceral adipose tissue in the belly secretes a variety of signaling molecules (adipokines) that are responsible for many of the effects related to obesity. ${ }^{12}$ Visceral or central adiposity is a stronger risk factor for EAC than is body mass index, ${ }^{5}$ and white men, who are more apt to have high central adiposity, have the highest incidence of EAC, relative to other races and women. ${ }^{30}$

Some adipokines have known effects in tumorigenesis and led us to analyze adipokines present in the abdominal fat pads to explain our observations of increased EAC tumor growth in obese mice. In particular, leptin, found to be higher in both the abdominal fat pads (Figure 4, $A$ and $B$ ) and serum (Figure 1,E) of HFD mice compared with CD mice, induces cellular proliferation and inhibits apoptosis of EAC cells in vitro. ${ }^{13,14}$ However, insulin growth factor-binding protein can induce tumor invasion and has been associated with worse patient prognosis because it suppresses oxidative stress-mediated cell death. ${ }^{36}$ Both TIMP1 and Serpin E1/PAI have well-documented protumorgeneic functions, regulating metastasis, growth, and survival $^{24-26}$ and were, interestingly, upregulated in the abdominal fat pads of HFD mice in the presence of the OE33 xenograft. This increased expression suggests possible cross-talk between the tumor and the abdominal fat of the HFD mice. More importantly, it highlights the fact that the effects of obesity and subsequent alterations in adipokines on EAC are complex, and additional studies are necessary to provide avenues toward opportunities for treatment in the clinical arena.

The past decade has brought increases in both EAC and obesity, in the United States and worldwide. ${ }^{3}$ In addition, the prevalence of obesity is not predicted to decrease in the near future, indicating a real need to understand the role of obesity in cancer. ${ }^{3}$ In this study, we find that a diet high in fat induces obesity in an immune-compromised mouse (NOD.SCID) and is correlated with an increased proliferation of human EAC xenograft tumors. Analysis of adipokine protein levels present in the abdominal fat pads of these mice revealed changes in several proteins within the obese mice. Several of these adipokines have relevance to tumorigenesis, and studies are ongoing within 
our laboratory to elucidate whether these proteins are involved in stimulation of OE33 growth. Likewise, studies directed toward the role of obesity and an HFD in EAC in more comparable models of Barrett's-esophagus-to-EAC progression are critical, as they provide a more reasonable platform to translate findings to the clinic. The recalcitrant nature of EAC, and the ever-increasing incidence of obesity, makes completion of these studies critical, as they are the first steps toward developing improved treatment for patients with EAC.

\section{Conflict of Interest Statement}

Ross Bremner reports consulting fees from Endostim. All other authors have nothing to disclose with regard to commercial support.

\section{References}

1. Siegel R, Naishadham D, Jemal A. Cancer statistics, 2013. CA Cancer J Clin. 2013;63:11-30

2. Brown LM, Devesa SS, Chow WH. Incidence of adenocarcinoma of the esophagus among white Americans by sex, stage, and age. J Natl Cancer Inst. 2008; 100:1184-7.

3. Ng M, Fleming T, Robinson M, Thomson B, Graetz N, Margono C, et al. Global, regional, and national prevalence of overweight and obesity in children and adults during 1980-2013: a systematic analysis for the Global Burden of Disease Study 2013. Lancet. 2014;384:766-81.

4. Simard EP, Ward EM, Siegel R, Jemal A. Cancers with increasing incidence trends in the United States: 1999 through 2008. CA Cancer J Clin. 2012;62: $118-28$.

5. Edelstein ZR, Farrow DC, Bronner MP, Rosen SN, Vaughan TL. Central adiposity and risk of Barrett's esophagus. Gastroenterology. 2007;133:403-11.

6. Vaughan TL, Kristal AR, Blount PL, Levine DS, Galipeau PC, Prevo LJ, et al. Nonsteroidal anti-inflammatory drug use, body mass index, and anthropometry in relation to genetic and flow cytometric abnormalities in Barrett's esophagus. Cancer Epidemiol Biomarkers Prev. 2002;11:745-52.

7. Ryan AM, Healy LA, Power DG, Byrne M, Murphy S, Byrne PJ, et al. Barrett esophagus: prevalence of central adiposity, metabolic syndrome, and a proinflammatory state. Ann Surg. 2008;247:909-15.

8. Nair S, Nguyen H, Salama S, Al-Hendy A. Obesity and the endometrium: adipocyte-secreted proinflammatory TNF alpha cytokine enhances the proliferation of human endometrial glandular cells. Obstetr Gynecol Int. 2013;2013: 368543.

9. Sundaram S, Freemerman AJ, Johnson AR, Milner JJ, McNaughton KK, Galanko JA, et al. Role of HGF in obesity-associated tumorigenesis: C3(1)TAg mice as a model for human basal-like breast cancer. Breast Cancer Res Treat. 2013;142:489-503.

10. Olivo-Marston SE, Hursting SD, Perkins SN, Schetter A, Khan M, Croce C, et al. Effects of calorie restriction and diet-induced obesity on murine colon carcinogenesis, growth and inflammatory factors, and microRNA expression. PloS One. 2014:9:e94765.

11. Coelho M, Oliveira T, Fernandes R. Biochemistry of adipose tissue: an endocrine organ. Arch Med Sci. 2013;9:191-200.

12. Moller DE, Kaufman KD. Metabolic syndrome: a clinical and molecular perspective. Annu Rev Med. 2005:56:45-62.

13. Ogunwobi O, Mutungi G, Beales IL. Leptin stimulates proliferation and inhibits apoptosis in Barrett's esophageal adenocarcinoma cells by cyclooxygenase-2dependent, prostaglandin-E2-mediated transactivation of the epidermal growth factor receptor and c-Jun NH2-terminal kinase activation. Endocrinology. 2006; 147:4505-16.
14. Somasundar P, Riggs D, Jackson B, Vona-Davis L, McFadden DW. Leptin stimulates esophageal adenocarcinoma growth by nonapoptotic mechanisms. Am J Surg. 2003;186:575-8.

15. Kendall BJ, Macdonald GA, Hayward NK, Prins JB, Brown I, Walker N, et al Leptin and the risk of Barrett's oesophagus. Gut. 2008;57:448-54.

16. Konturek PC, Burnat G, Rau T, Hahn EG, Konturek S. Effect of adiponectin and ghrelin on apoptosis of Barrett adenocarcinoma cell line. Dig Dis Sci. 2008;53: 597-605.

17. Boonstra JJ, van Marion R, Beer DG, Lin L, Chaves P, Ribeiro C, et al. Verification and unmasking of widely used human esophageal adenocarcinoma cell lines. J Natl Cancer Inst. 2010;102:271-4.

18. Van Heek M, Compton DS, France CF, Tedesco RP, Fawzi AB, Graziano MP et al. Diet-induced obese mice develop peripheral, but not central, resistance to leptin. J Clin Invest. 1997;99:385-90.

19. Tomayko MM, Reynolds CP. Determination of subcutaneous tumor size in athymic (nude) mice. Cancer Chemother Pharmacol. 1989;24:148-54.

20. Whitsett TG, Fortin Ensign SP, Dhruv HD, Inge LJ, Kurywchak P, Wolf KK, et al FN14 expression correlates with MET in NSCLC and promotes MET-driven cell invasion. Clin Exp Metastasis. 2014;31:613-23.

21. Abnet CC, Freedman ND, Hollenbeck AR, Fraumeni JF Jr, Leitzmann M, Schatzkin A. A prospective study of BMI and risk of oesophageal and gastric adenocarcinoma. Eur J Cancer. 2008;44:465-71.

22. Alexander J, Chang GQ, Dourmashkin JT, Leibowitz SF. Distinct phenotypes of obesity-prone AKR/J, DBA2J and C57BL/6J mice compared to control strains. Int J Obes (Lond). 2006;30:50-9.

23. Zhou H, Luby-Phelps K, Mickey BE, Habib AA, Mason RP, Zhao D. Dynamic near-infrared optical imaging of 2-deoxyglucose uptake by intracranial glioma of athymic mice. PloS One. 2009;4:e8051.

24. Andreasen PA. PAI-1 - a potential therapeutic target in cancer. Curr Drug Targets. 2007;8:1030-41.

25. Chirco R, Liu XW, Jung KK, Kim HR. Novel functions of TIMPs in cell signaling. Cancer Metastasis Rev. 2006;25:99-113.

26. Fang H, Placencio VR, DeClerck YA. Protumorigenic activity of plasminogen activator inhibitor-1 through an antiapoptotic function. $J$ Natl Cancer Inst. 2012;104:1470-84

27. Watanabe R, Tomita N, Takeuchi K, Sakata S, Tateishi U, Tanaka M, et al. SUVmax in FDG-PET at the biopsy site correlates with the proliferation potential of tumor cells in non-Hodgkin lymphoma. Leuk Lymphoma. 2010;51:279-83.

28. Ekmekcioglu O, Aliyev A, Yilmaz S, Arslan E, Kaya R, Kocael P, et al. Correlation of 18F-fluorodeoxyglucose uptake with histopathological prognostic factors in breast carcinoma. Nucl Med Commun. 2013;34:1055-67.

29. Mai V, Colbert LH, Berrigan D, Perkins SN, Pfeiffer R, Lavigne JA, et al. Calorie restriction and diet composition modulate spontaneous intestinal tumorigenesis in Apc(Min) mice through different mechanisms. Cancer Res. 2003;63:1752-5.

30. Reid BJ, Li X, Galipeau PC, Vaughan TL. Barrett's oesophagus and oesophagea adenocarcinoma: time for a new synthesis. Nat Rev Cancer. 2010;10:87-101.

31. Yoshimoto S, Loo TM, Atarashi K, Kanda H, Sato S, Oyadomari S, et al. Obesityinduced gut microbial metabolite promotes liver cancer through senescence secretome. Nature. 2013;499:97-101.

32. Ballak DB, Stienstra R, Hijmans A, Joosten LA, Netea MG, Tack CJ. Combined B- and T-cell deficiency does not protect against obesity-induced glucose intolerance and inflammation. Cytokine. 2013;62:96-103.

33. Quante M, Bhagat G, Abrams JA, Marache F, Good P, Lee MD, et al. Bile acid and inflammation activate gastric cardia stem cells in a mouse model of Barrettlike metaplasia. Cancer Cell. 2012;21:36-51.

34. Wang J, Myles B, Wei C, Chang JY, Hofstetter WL, Ajani JA, et al. Obesity and outcomes in patients treated with chemoradiotherapy for esophageal carcinoma. Dis Esophagus. 2014:27:168-75.

35. Dignam JJ, Polite BN, Yothers G, Raich P, Colangelo L, O'Connell MJ, et al Body mass index and outcomes in patients who receive adjuvant chemotherapy for colon cancer. J Natl Cancer Inst. 2006;98:1647-54.

36. Natsuizaka M, Kinugasa H, Kagawa S, Whelan KA, Naganuma S, Subramanian H, et al. IGFBP3 promotes esophageal cancer growth by suppressing oxidative stress in hypoxic tumor microenvironment. Am J Cancer Res. 2014; 4:29-41. 